\title{
Migración de dispositivo intrauterino a vejiga: Aportación de cuatro casos
}

\author{
Ebel L, Foneron A, Troncoso L, Cañoles R, Hornig A, Corti D.
}

\begin{abstract}
Unidad de Urología. Universidad Austral de Chile. Servicio de Urología. Hospital Regional de Valdivia. Departamento de Urología. Universidad Austral de Chile.
\end{abstract}

Actas Urol Esp. 2008;32(5):530-532

\section{RESUMEN}

MIGRACIÓN DE DISPOSITIVO INTRAUTERINO A VEJIGA: APORTACIÓN DE CUATRO CASOS

El dispositivo intrauterino (DIU) ha sido utilizado en nuestro medio durante muchos años como método anticonceptivo. Una de las complicaciones es la migración fuera del útero, siendo la localización vesical inusual, con escasos casos en la literatura. Presentamos cuatro casos de migración del DIU a la vejiga. Se describe cuadro clínico, estudio y tratamiento.

Palabras claves: Dispositivo intrauterino. Litiasis vesical. Perforación uterina.

\section{ABSTRACT}

\section{INTRAUTERINE DEVICE MIGRATION TO THE BLADDER: FOUR CASES REPORT}

The intrauterine device has been commonly used in our population for many years as contraceptive method. One of the complications is the device migration out of the uterus. One of the most uncommon fait of this migration is the bladder, with few reports on the literature. In this work, four cases are reported with their clinical presentation, study and treatment.

Keywords: Intrauterine devices. Bladder stones. Uterine Perforation.

$\mathrm{E}$ dispositivo intrauterino (DIU) es uno de los métodos anticonceptivos más usados debido a su alta eficacia, bajo riesgo y bajo costo. Su uso tiene escasas complicaciones, entre las cuales destacan la perforación uterina (1-3 x 1000 inserciones), hemorragias, dolor, abortos sépticos, embarazos ectópicos y migración. La perforación se produce habitualmente por una mala técnica de inserción y provoca dolor durante el procedimiento ${ }^{1,2}$. El riesgo de perforación uterina aumenta en el puerperio o fuera de la menstruación y predispone a la migración del DIU. Habitualmente ocurre a regiones adyacentes a los órganos ginecológicos, sin embargo se han descrito casos de DIU en peritoneo, omento, apéndice y colon ${ }^{3-5}$.

Cuando el DIU migra a la vejiga, lo que es inusual, esto se manifiesta habitualmente con síntomas y signos como polaquiuria, tenesmo, dolor suprapúbico, disuria, hematuria, infección urinaria, obstrucción urinaria por litiasis, incontinencia de orina ${ }^{6,7}$. Lo más frecuente es que se manifieste como infección urinaria persistente o recurrente, siendo por lo general el diagnóstico de DIU intravesical un hallazgo durante el estudio ${ }^{8-11}$. En la mayoría de los casos están asociados a cálculos vesicales secundarios $^{12-20}$.

\section{PACIENTES Y MÉTODOS}

En nuestro servicio, durante los últimos 30 años, se han presentado 147 pacientes con diagnóstico de litiasis vesical de los cuales un $15 \%$ (22/147) eran mujeres. De estas, un 19\% tenía un DIU intravesical (4 casos).

La edad promedio de nuestra serie al momento del diagnóstico fue de 33 años (28-37). El intervalo entre la inserción del DIU y los sintomas urinarios es variable. En nuestras pacientes se presentó, en promedio, 10,3 años después de la colocación del DIU. La forma de presentación más frecuente fue un síndrome de irritación vesical. Tres pacientes tenían infección urinaria y dos pacientes hematuria. Todas presentaban cálculos vesicales asociados. Destacaba que 2 pacientes se habían embarazado, una 6 años y la otra 7 años después de insertado el DIU (Tabla 1). 
Tabla 1. Tabla Resumen

\begin{tabular}{|c|c|c|c|c|c|c|c|c|}
\hline & \multirow[b]{2}{*}{ Edad (años) } & \multirow[b]{2}{*}{$\begin{array}{c}\text { Intervalo } \\
\text { sintomas } \\
\text { (años) }\end{array}$} & \multicolumn{6}{|c|}{ Síntomas } \\
\hline & & & $\begin{array}{l}\text { Irritación } \\
\text { vesical }\end{array}$ & ITU & Dolor & Hematuria & $\begin{array}{l}\text { Litiasis } \\
\text { vesical }\end{array}$ & $\begin{array}{c}\text { Embarazo } \\
\text { con DIU }\end{array}$ \\
\hline Caso 1 & 28 & 7 & $\mathrm{X}$ & $\mathrm{X}$ & & $\mathrm{X}$ & $\mathrm{X}$ & \\
\hline Caso 2 & 37 & 10 & $\mathrm{X}$ & $\mathrm{X}$ & $\mathrm{X}$ & & $\mathrm{X}$ & $\mathrm{X}$ \\
\hline Caso 3 & 34 & 7 & $\mathrm{X}$ & $\mathrm{X}$ & & & $\mathrm{X}$ & $\mathrm{X}$ \\
\hline Caso 4 & 34 & 17 & $\mathrm{X}$ & & & $\mathrm{X}$ & $\mathrm{X}$ & \\
\hline Promedio & 33 & 10,3 & & & & & & \\
\hline
\end{tabular}

En las primeras tres pacientes se llegó al diagnóstico durante el estudio de infecciones urinarias recurrentes, sospechándose DIU intravesical en la pielografía de eliminación (Figs. 1 y 2). En una

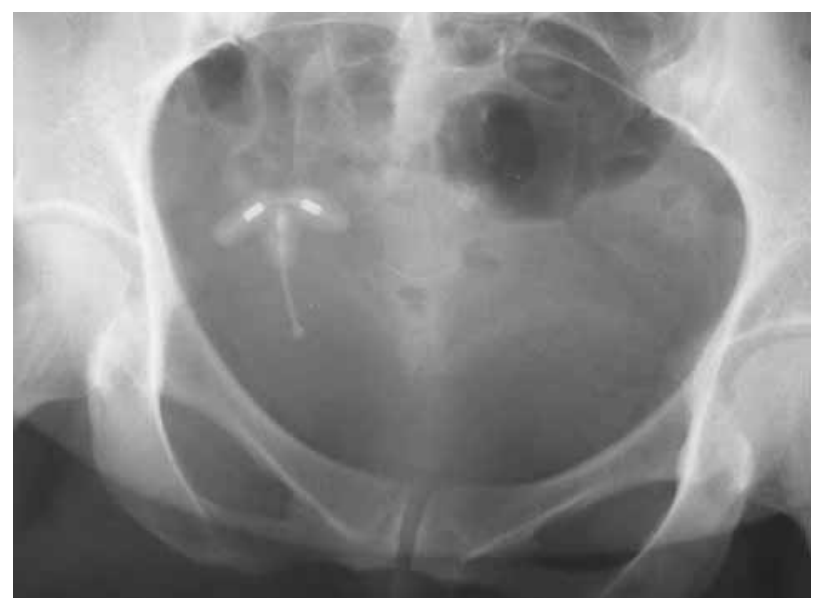

FIGURA 1. Caso 1. Radiografia vesical simple. Se observa DIU calcificado en zona vesical.

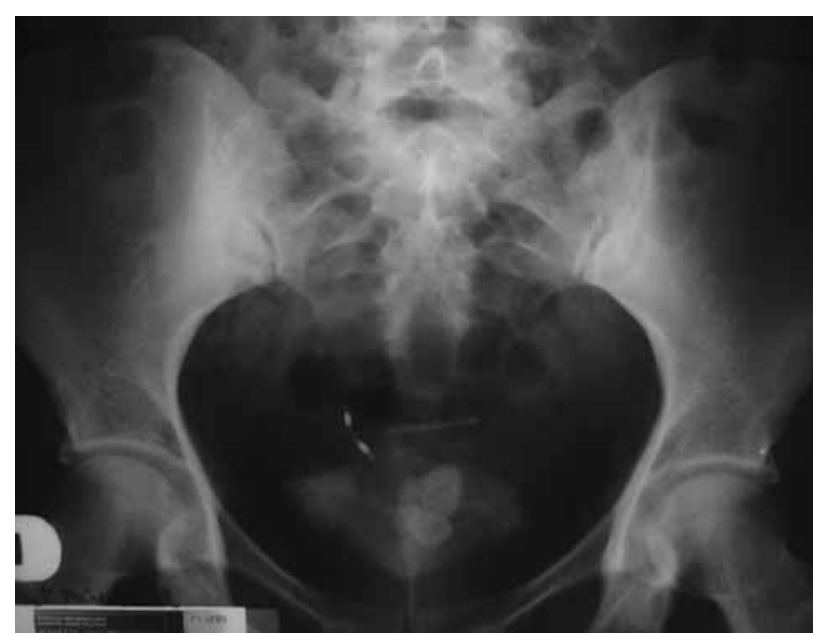

FIGURA 2. Caso 2. Radiografia postmiccional de pielografia de eliminación. Se observan cálculos vesicales y DIU intimamente adherido a pared vesical. paciente fue en un estudio ecográfico en que se sospechó un DIU ectópico. Todas fueron confirmadas con cistoscopía (Fig. 3).

En la mayoría de los casos el tratamiento ha sido endóscopico. En nuestro servicio el tratamiento en los cuatro casos fue cistolitotomía suprapúbica con retiro del DIU (Fig. 4). Todas evolucionaron sin complicaciones. En una de las pacientes no fue posible extraer el DIU completo por no encontrarse parte del vástago. Dos años después presentó nuevamente cálculos vesicales asociado a trozo de DIU, requiriendo una nueva intervención.

Con un seguimiento promedio de 3,5 años (2-7 años) nuestras pacientes no han presentado nuevas recidivas de litiasis vesical o infecciones urinarias.

\section{CONCLUSIÓN}

La migración del DIU a vejiga parece ser una complicación de baja frecuencia, sin embargo cuando nos enfrentamos a mujeres con síntomas de irri-

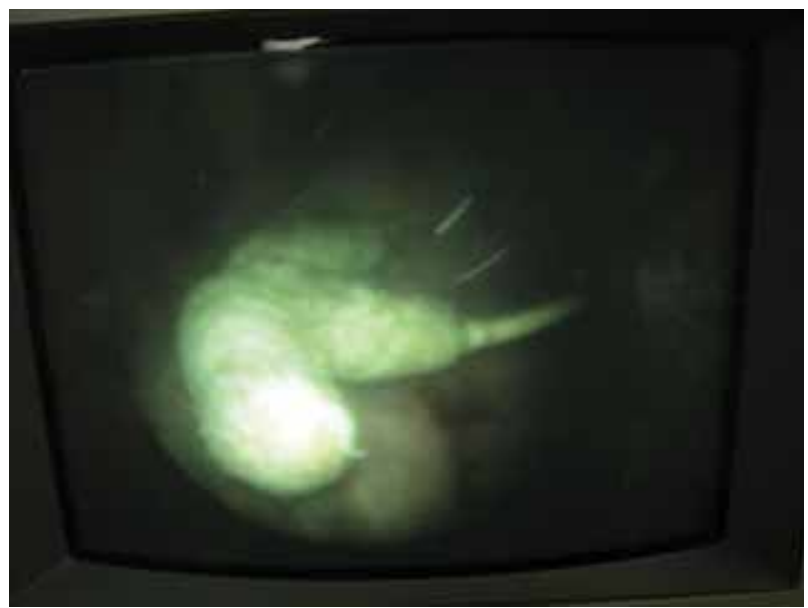

FIGURA 3. Caso 1. Cistoscopia, donde se observa DIU calcificado intravesical. 


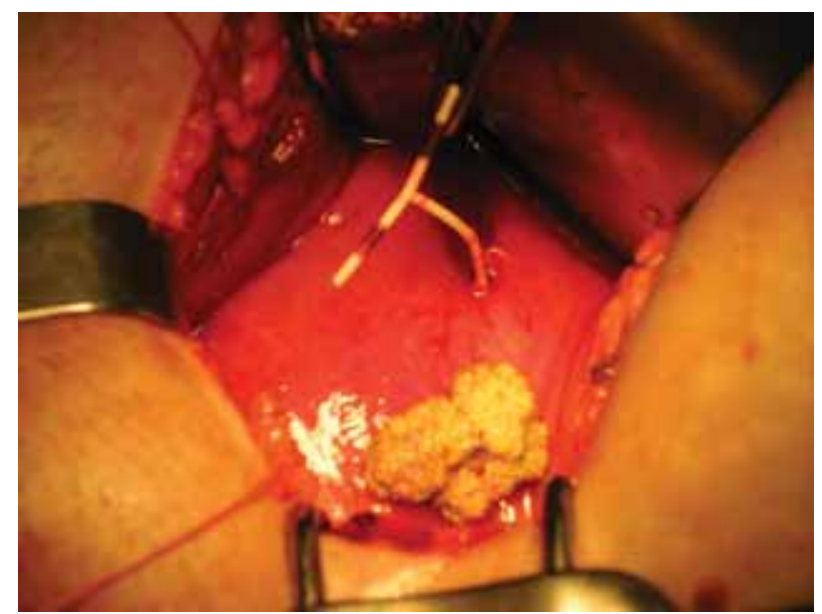

FIGURA 4. Caso 2. Imagen intraoperatoria a través de cistotomia. Se observa DIU atravesando la pared vesical y cálculos vesicales.

tación vesical, infecciones urinarias recurrentes o persistentes y aún más, en mujeres con litiasis vesical, se debe considerar entre los diagnósticos diferenciales. La pielografía de eliminación es habitualmente el primer examen donde se sospecha esta entidad, que deberá ser confirmada mediante cistoscopia. El tratamiento endoscópico es el adecuado siempre que logre retirar el DIU en su totalidad. Si esto no es posible se debe realizar una cirugía abdominal.

\section{REFERENCIAS}

1. Caliskan E, Ozturk N, Dilbaz BO, Dilbaz S. Analysis of risk factors associated with uterine perforation by intrauterine devices. Europ J Contracept Reprod Health Care 2003;8(3):150-155.

2. Zakin D, Stern WZ, Rosenblatt R. Complete and partial uterine perforation and embedding following insertion of intrauterine devices. I: Classification, complications, mechanism, incidence and missing string. Obstet Gynecol Surv. 1981;36(7):335-353.

3. Neumann T, López J, Pardo M. Extracción de dispositivos intrauterinos de localización intestinal por laparoscopía. Rev Chil Obstet Ginecol. 1999;64(3):209-211.

4. Nceboz US, Ozcakir HT, Uyar Y, Caglar H. Migration of an intrauterine contraceptive device to the sigmoid colon: a case report. Eur J Contracept Reprod Health Care. 2003;8(4):229232.

5. Disu S, Boret A. Asymptomatic ileal perforation of an intrauterine device. Arch Gynecol Obstet. 2004;269(3):230-231.

6. Pascual Regueiro D, García de Jalón Martínez A, Mallén Mateo E, Sancho Serrano C, Gonzalvo Ibarra A, Rioja Sanz LA. Intravesical foreign bodies. Review of the literature. Actas Urol Esp. 2003;27(4):265-273.
7. Yalcin V, Demirkesen O, Alici B, Onol B, Solok V. An unusual presentation of a foreign body in the urinary bladder: a migrant intrauterine device. Urol Int. 1998;61(4):240-242.

8. Ruiz M, Soto E, Rioseco E, Campodonico I. Dispositivo intrauterino (T-cu). Diagnóstico ecografico. Rev Chil Obstet Ginecol. 1979;44(3):84-88.

9. McNamara M, Kennan N, Buckley AR. Copper-7 perforation of the uterus and urinary bladder with calculus formation-sonographic demonstration. Br J Radiol. 1985;58(690):558-559.

10. Sepulveda WH, Ciuffardi I, Olivari A, Gallegops O. Sonographic diagnosis of bladder perforation by an intrauterine device. J Reprod Med. 1993;38(11):911-913.

11. Ozcelik B, Serin IS, Basbug M, Aygen E, Ekmekcioglu O. Differential diagnosis of intra-uterine device migrating to bladder using radiographic image of calculus formation and review of literature. Eur J Obstet Gynecol Reprod Biol. 2003;108(1): 94-96.

12. Soronwala K, Singh R, Dass H. Lippes loop perforation of the uterus and urinary bladder with stone formation. Obstet Ginecol. 1974;44(3):424-427.

13. Iglesias J, Corbalan G, Galan G, Gayan P. Perforación uterina y de vejiga urinaria con asa de Lippes. Rev Chil Obstet Ginecol. 1977;42(3): 149-150.

14. Cumming GP, Bramwell SP, Lees DA. An unusual case of cystolithiasis: a urological lesson for gynaecologists. Br J Obstet Gynecol. 1997;104(1):117-118.

15. Lu HF, Chen JH, Chen WC, Shen WC. Vesicle calculus caused by migrant intrauterine device. AJR Am J Roentgenol. 1999; 173(2):504-505.

16. Guvel S, Tekin MI, Kilinc F, Peskircioglu L, Ozkardes H. Bladder stones around a migrated and missed intrauterine contraceptive device. Int J Urol. 2001;8(2):78-79.

17. Salazar A, Orellana N, Cortés M, Montiglio C. Litiasis vesical secundaria a dispositivo intrauterino incrustado en pared vesical: Revisión de dos casos clínicos. Rev Chil Urol 2002;67:8586.

18. Nwofor AM, Ikechebelu JI. Uterovesical fistula and bladder stones following bladder penetration by a perforating intrauterine contraceptive device. J Obstet Gynaecol. 2003;23(6): 683-684.

19. Rafique M, Rauf A, Khan NA, Haque TU. An unusual cause of vesical stone: a migrant intrauterine device. Eur J Contracept Reprod Health Care. 2003;8(3):170-172.

20. Hick E, Hernandez J, Yordan R, Money AF, Aviles R, Garcia CR. Bladder calculus resulting from the migration of an intrauterine contraceptive device. J Urol. 2004;172(5 Pt 1):1903.

Correspondencia autor: L.F. Ebel Sepulveda Servicio de Urología. Hospital Regional de Valdivia Bueras esq. Bulnes, Chile Tel.: 63-297150

E-mail autor: Luisebel@uach.cl

Información artículo: Original - Urología general Trabajo recibido: julio 2007

Trabajo aceptado: noviembre 2007 